\title{
Predictive value of the glycated albumin versus glycosylated hemoglobin in follow-up of glucose homeostasis in hemodialysis-maintained type-2 diabetic patients
}

\author{
Elham Ahmed ${ }^{1}$, Fatma EL Zahra A SAyed Bokhary ${ }^{1}$, Samar Ismail ${ }^{1}$, \\ Waleed Mahmoud Abd EL Hameed ${ }^{2}$
}

\begin{abstract}
${ }^{1}$ Internal Medicine Department, Faculty of Medicine, Minia University, Minia, Egypt; ${ }^{2}$ Clinical Pathology Department, Faculty of Medicine, Minia University, Minia, Egypt

E-mail:waleed_mahmoud72@yahoo.com
\end{abstract}

\begin{abstract}
Objectives. Markers for glucose control in hemodialysis patients (HDP) are debated. Glycosylated hemoglobin $(\mathrm{HbAlc} \%)$ relies on the stable red blood cell survival. Albumin turnover is faster than hemoglobin. Glycated albumin (GA\%) may be used as an index of short-term glycemic control. The predictive value of GA\% versus $\mathrm{HbAlc} \%$ in monitoring the glucose homeostasis in type-2 diabetic HDP is studied.

Methods. Forty type- 2 diabetic HDP and 20 healthy non diabetic subjects matched age and sex as a control group were included. Calculation of body mass index and Homeostatic Model Assessment of Insulin Resistance (HOMA-IR) and urea reduction ratio were done. Glycosylated hemoglobin, glycated albumin, fasting blood glucose, insulin, total lipid, kidney and liver functions tests, hepatitis markers, electrolytes, complete blood count, and international normalized ratio were performed. Patients were followed up after 6 months.

Results. The study showed that GA\% is more sensitive than HbA1c\%, but less specific in the follow-up of the glucose homeostasis in type-2 diabetic HDP. Diagnostic accuracy is higher in $\mathrm{HbAlc} \%$ than in GA\%. HOMA-IR is superior regarding the sensitivity and the diagnostic accuracy.

Conclusion. The present data show that GA\% is more sensitive than $\mathrm{HbAlc} \%$ and has more diagnostic accuracy in the follow-up of the glucose homeostasis in type-2 diabetic HDP.
\end{abstract}

Key words: glycated albumin, glycosylated hemoglobin, glucose homeostasis, hemodialysis, type-2 diabetes

Accurate assessment of glycemic control is vital to improve the outcome and survival of hemodialysis-maintained diabetic patients. The best glycemic marker for glucose control in these patients is still debated. This is because several clinical and pharmacological factors that may affect the ability of the available biomarkers to reflect the patient's glycemic status properly (Abe et al. 2019). It is controversial whether glycosylated hemoglobin (HbAlc\%) accurately reflects the mean blood glucose levels in diabetic patients maintained on hemodialysis, as some would argue that it may not be a reliable marker for a long-term glycemic control. Glycated albumin (GA\%), which reflects glycemic control over a shorter period, may be of greater value for predicting the clinical outcomes in hemodialysis patients. It might be a marker for monitoring glycemic control in diabetic patients with end-stage renal disease (ESRD) (Abe et al. 2018; ADA 2018). In cases with ESRD, the renal anemia lowers the $\mathrm{HbAlc} \%$ values because the 
lifespan of the erythrocytes is shortened. However, in patients with diabetic nephropathy with marked proteinuria, the GA\% values are lower because of the increased turnover of the albumin metabolism (Abe et al. 2018).

The aim of the study was to investigate the value of $\mathrm{GA} \%$, compared to $\mathrm{HbA} 1 \mathrm{c} \%$, in monitoring the glycemic status in hemodialysis (HD)-maintained patients with type-2 diabetes mellitus.

\section{Subjects and methods}

Subjects. Our study was prospective case-control that involved 40 type-2 diabetic patients on regular hemodialysis recently (less than one-year duration) after giving written consent. Patients were selected from the Minia University Hemodialysis Unit. This research was permitted by the Institutional Ethics Committee of the Faculty of Medicine, Minia University, Egypt. This research was directed with the ethical guidelines in the 1975 Helsinki declaration. Diabetes was diagnosed according to the criteria of the American Diabetes Association (ADA), where a patient is considered to be diabetic when fasting glucose is more than or equal to $126 \mathrm{mg} / \mathrm{dL}$ $(7.0 \mathrm{mmol} / \mathrm{L})$ or 2 -h plasma glucose is more than or equal to $200 \mathrm{mg} / \mathrm{dL}(11.1 \mathrm{mmol} / \mathrm{L})$ next to a $75 \mathrm{~g}$ oral glucose test or a patient had HbA1C more than $6.5 \%$ (Aramwit and Satirapoj 2013). This study involved 20 healthy persons as a control group of the same age and sex. This study excluded patients with type-1 diabetes mellitus (DM), conditions affecting blood homeostasis as acute and chronic blood loss, recent blood transfusion, hemolytic anemia, splenomegaly, haemoglobinopathies, liver cirrhosis, HIV, or alcohol abuse.

Full history was taken from our patients, particularly the history of smoking, duration of diabetes, drugs (oral hypoglycemic drugs/insulin), history of diabetic complication (recurrent infections, retinopathy and diabetic foot), hypertension or presence of cardiovascular disease (CVD) (defined as previous myocardial or cerebral infarction), current drugs for hypertension or hyperlipidemia, erythropoietin stimulating agents, and hemodialysis history (duration of HD, dry weight, UF volume and HD complication).

Parameters measured. Calculation of body mass index (BMI) was done according to the data of the National Institute of Health: BMI = weight $(\mathrm{kg}) /$ height $\left(\mathrm{m}^{2}\right)$ (dry weight in HD patients). Obesity was determined when BMI was more than $30 \mathrm{~kg} /$ $\mathrm{m}^{2}$. Detection of HOMA-IR (Homeostatic Model Assessment of Insulin Resistance) to all groups where normal range was (0.5-1.4), less than 1.0 means optimal insulin sensitivity, over 1.9 means early insulin resistance, and more than 2.9 means significant insulin resistance (Buell et al. 2007).

HOMA-IR was calculated by the following equation: fasting insulin $(\mathrm{micro} / \mathrm{L}) \times$ fasting glucose $(\mathrm{mmol} / \mathrm{L})$ (Buell et al.2007). Detection of the adequacy of hemodialysis was done by the following equation: urea reduction ratio $(\mathrm{URR})=(\mathrm{U}$ pre $-\mathrm{U}$ post $/ \mathrm{U}$ pre $)$ $\times 100 \%$, where $\mathrm{U}$ pre is the pre-dialysis urea level and $\mathrm{U}$ post is the post-dialysis urea level. The URR is the urea decrease percentage; practically it is multiplied by $100 \%$ and stated as a ratio (Campbell 1988). Echoduplex study was performed to detect the ejection fraction of patients and the control group at the start of study.

Hemoglobin A1c\% was measured utilizing boronate affinity by Nyco-Card READER II (Cefalu et al. 2017). Glycated albumin (GA) was measured using sandwich ELISA detection method. Reference range of glycated albumin is (0.4-2\%) (Chen et al. 2017). Fasting blood glucose, kidney functions tests, total lipid profile, serum sodium, calcium, potassium and viral markers (HCV Abs, HBs Ag, HIV Abs) were tested by fully automated ChemiLuminescence technology (Cobas E 411-Roche-Roche Diagnostics GmbH Germany). Complete blood count was performed with an automated cell counter (Sysmex KX-21N, TAO Medical Incorporation, Japan). The international normalized ratio (INR) was detected by a STAGO COMPACT CT Coagulation Analyzer (Diamond Diagnostics, USA); HC Abs, HBsAg and HIV Abs were detected with fully automated ChemiLuminescence technology (Cobas E 411-RocheRoche Diagnostics $\mathrm{GmbH}$ Germany). Fasting serum insulin was assayed by a double-antibody immunochemi-luminometric method completed on an Access Automated Platform (Beckman Instruments, Chaska, MN). Serum insulin normal level was 0.7-9.0 $\mu \mathrm{U} / \mathrm{ml}$; diabetic level (Type 2) was 0.7-25 $\mu \mathrm{U} /$ $\mathrm{ml}$ (Cohen and Hud 1989). The follow-up study was done by comparison of patients' group data at start of study and after 6 months of regular hemodialysis as regards clinical, laboratory and insulin resistance (HOMA-IR). The patients' groups were further subdivided into controlled and uncontrolled groups according to presence or absence of diabetic complications at time of inclusion in the study. Comparison between clinical and laboratory data between both subgroups was done at start of study and after 6-month follow-up.

Statistical analysis. The SPSS program (Statistical Package for Social Sciences) software version 
25 was used statistical analysis of data. Descriptive statistics were done for parametric quantitative data by mean $\pm S D$, and for non-parametric quantitative data by median and interquartile range (IQR), while they were done for categorical data by number and percentage. Correlations among changed variables were found utilizing Pearson's and Spearman's correlation coefficients. Simple logistic regression test was used for the prediction of uncontrolled DM. The ROC (receiver operating characteristic) curve analysis was used to detect AUC, optimal cut-off point, sensitivity, specificity, positive predictive value (PPV), negative predictive value (NPV) and diagnostic accuracy of the studied markers useful in the prediction of uncontrolled DM and difference between glycated albumin and Hbalc\% in such issue. The significant p-value was less than 0.05 .

\section{Results}

Our study showed that the patients' group had significant higher BMI than the control group with median duration of DM about 15 years. Fifty-five percent of them had diabetic complications. About $62.2 \%$ of the patients were hypertensive with a median duration of 12 years. As regards hemodialysis complications, $38.5 \%$ of the patients had developed complications such as intradialytic hypotension (17.5\%), intra-dialytic hypoglycemia (17.5\%), and cramps (2.5\%); the median duration of hemodialysis was 3 months (at start of study) and the mean erythropoietin dosage was 2.6. The patients' group had significant lower hemoglobin level, platelets count and serum albumin $(\mathrm{p}<0.001)$ in addition to higher serum creatinine level $(\mathrm{p}<0.001)$. Fifteen percent of the patients' group had positive HCV antibodies. The patients' group showed highly significant increased $\mathrm{HbAlc} \%$, GA\%, fasting blood glucose, fasting insulin and HOMA-IR compared to the control group $(\mathrm{p}<0.001)$ (Table 1$)$.

$\mathrm{HbAlc} \%$, GA\%, and serum urea were significantly lower after 6-month follow-up than at start of study $(\mathrm{p}<0.001)$. The platelet count, serum fasting insulin and URR were significantly higher after 6-month follow-up than that at start of study $(\mathrm{p}<0.001)$. Serum albumin was higher in the follow-up data than at start of study $(\mathrm{p}<0.04)$ (Table 2). According to the baseline data at start of study, there were statistically insignificant differences between controlled and uncontrolled groups as regards $\mathrm{Hb} \%$, creatinine, urea level, URR, serum albumin level. Only total leucocytic count (TLC) had showed significant difference between both groups $(\mathrm{p}<0.03)$ (Table 3$)$.
HbAlc\%, GA\% and HOMA-IR after 6-month follow-up were significantly lower in the controlled group than the uncontrolled with $(\mathrm{p}<0.023, \mathrm{p}<0.015$ and $\mathrm{p}<0.001)$, respectively. Total cholesterol after 6-month follow-up was significantly lower in the controlled group than the uncontrolled $(\mathrm{p}<0.027)$ (Table 4).

There was highly significant correlation between each of HbAlc\% and glycated albumin (GA) and fasting blood sugar (FBS), total cholesterol, and HOMA-IR $(\mathrm{p}<0.001), \mathrm{Hb}(\mathrm{p}<0.024)$ and triglycerides $(\mathrm{p}<0.008)$. No significant correlation between $\mathrm{HbAlc} \%$ and erythropoietin dose or URR (Table 5).

After 6-month follow-up, there was highly significant correlation between $\mathrm{HbAlc} \%$ and $\mathrm{Hb}$, fasting insulin, HOMA-IR $(\mathrm{p}<0.001)$, and TLC $(\mathrm{p}<0.003)$. There was also highly significant association between GA with $\mathrm{HbAlc} \%$ and fasting insulin, HOMA-IR $(\mathrm{p}<0.001), \mathrm{Hb}(\mathrm{p}<0.008)$, TLC with $(\mathrm{p}<0.034)$, and total cholesterol $(\mathrm{p}<0.023)$ (Table 6).

Table 7 reveals that the most predicted variable is HOMA-IR as the increase of HOMA-IR by one unit will increase the risk of uncontrolled DM by $190 \%$ $(\mathrm{OR}=2.9,95 \% \mathrm{CI}=1.21-6.95)$, followed by $\mathrm{GA}$ as its increase by one unit will increase the risk of uncontrolled DM by $186 \%(\mathrm{OR}=2.86,95 \% \mathrm{CI}=1.17-7.04)$, then HBAlc as its increase by one unit will increase the risk by $77 \%(\mathrm{OR}=1.77,95 \% \mathrm{CI}=1.04-3.02)$, then TLC as its increase by one unit will increase the risk by $56 \%(\mathrm{OR}=1.56,95 \% \mathrm{CI}=1.13-2.16)$ and lastly total cholesterol (TC) as its increase by one unit will increase the risk by $1 \%(\mathrm{OR}=1.01,95 \% \mathrm{CI}=1.01-1.03)$; however, the increase in urea will decrease the risk by $6 \%$ (OR=0.94, 95\% CI=0.9-0.99).

ROC curve analysis of HOMA-IR revealed that AUC $=0.831$ optimal cut-off $\geq 3.4$ with sensitivity $100 \%$, specificity $66.67 \%$ and accuracy $85 \%$. ROC curve analysis of $\mathrm{HbAlc}$ revealed that $\mathrm{AUC}=0.768$ optimal cut off $\geq 4.6$ with sensitivity $86.36 \%$, specificity $61.11 \%$ and accuracy $75 \%$. ROC curve analysis of GA revealed that $\mathrm{AUC}=0.730$ optimal cut off $\geq 2.0$ with sensitivity $90.91 \%$, specificity $50 \%$ and accuracy $72.5 \%$ (Table 8).

\section{Discussion}

$\mathrm{HbAlc} \%$ measurement relies on a relatively stable RBC survival, which is characteristic for general population, but not patients on HD (Divani et al. 2018). The metabolic turnover of albumin is faster than hemoglobin, with a lifespan of 17 to 23 days. So, GA\% is used as an index of short-term glycemic control. GA is more closely associated with the 
Table 1

Demographic, clinical and laboratory characteristics of the studied subjects.

\begin{tabular}{|c|c|c|c|c|}
\hline Variables & & $\begin{array}{c}\text { Patients group } \\
(\mathrm{n}=40)\end{array}$ & $\begin{array}{c}\text { Control group } \\
(n=20)\end{array}$ & p-value \\
\hline Age (years) & $\begin{array}{l}\text { Range } \\
\text { Mean } \pm S D\end{array}$ & $\begin{array}{c}55-75 \\
62.5 \pm 5.1\end{array}$ & $\begin{array}{c}20-40 \\
27.9 \pm 5.4\end{array}$ & $<0.001^{\star}$ \\
\hline Gender & $\begin{array}{l}\text { Male } \\
\text { Female }\end{array}$ & $\begin{array}{c}8(20 \%) \\
32(80 \%)\end{array}$ & $\begin{array}{c}8(40 \%) \\
12(60 \%)\end{array}$ & 0.099 \\
\hline Weight (kg) & $\begin{array}{l}\text { Range } \\
\text { Mean } \pm S D\end{array}$ & $\begin{array}{c}58-107 \\
71.1 \pm 10.1\end{array}$ & $\begin{array}{c}55-75 \\
62.9 \pm 5.4\end{array}$ & $<0.001^{*}$ \\
\hline BMI $\left(\mathrm{kg} / \mathrm{m}^{2}\right)$ & $\begin{array}{l}\text { Range } \\
\text { Mean } \pm S D\end{array}$ & $\begin{array}{c}22.2-38.0 \\
27.3 \pm 3.3\end{array}$ & $\begin{array}{l}20.2-28.0 \\
24.3 \pm 2.04\end{array}$ & $<0.001^{*}$ \\
\hline DM duration (years) & Median (IQR) & $15(10.5-18.0)$ & & \\
\hline DM treatment & $\begin{array}{l}\text { Oral } \\
\text { Insulin }\end{array}$ & $\begin{array}{c}7(17.5 \%) \\
33(82.5 \%)\end{array}$ & & \\
\hline Hypertension (HTN) & $\begin{array}{l}\text { No } \\
\text { Yes }\end{array}$ & $\begin{array}{l}15(37.5 \%) \\
25(62.5 \%)\end{array}$ & & \\
\hline DM complications & $\begin{array}{l}\text { No } \\
\text { Yes }\end{array}$ & $\begin{array}{l}18(45 \%) \\
22(55 \%)\end{array}$ & & \\
\hline HTN duration (years) & Median (IQR) & $12(10-15)$ & & \\
\hline Ejection fraction (EF\%) & $\begin{array}{l}\text { Normal } \\
\text { Impaired }\end{array}$ & $\begin{array}{c}35(87.5 \%) \\
5(12.5 \%)\end{array}$ & Normal (100\%) & 0.089 \\
\hline Erythropoietin dosage & $\begin{array}{l}\text { Range } \\
\text { Mean } \pm S D\end{array}$ & $\begin{array}{c}2-3 \\
2.6 \pm 0.5\end{array}$ & & \\
\hline Hemodialysis complications & $\begin{array}{l}\text { No } \\
\text { Yes }\end{array}$ & $\begin{array}{l}25(62.5 \%) \\
15(38.5 \%)\end{array}$ & & \\
\hline $\mathrm{Hb}(\mathrm{gm} / \mathrm{dl})$ & $\begin{array}{l}\text { Range } \\
\text { Mean } \pm S D\end{array}$ & $\begin{array}{c}7-11 \\
9.3 \pm 1.0\end{array}$ & $\begin{array}{c}12-15 \\
13.2 \pm 0.8\end{array}$ & $<0.001^{*}$ \\
\hline $\operatorname{TLC}\left(\mathrm{x} 10^{9} / \mathrm{L}\right)$ & Median (IQR) & $7(5.9-9.0)$ & $6.3(4.9-6.9)$ & $0.037^{*}$ \\
\hline Platelets (x109/L) & $\begin{array}{l}\text { Range } \\
\text { Mean SD }\end{array}$ & $\begin{array}{c}120-254 \\
166.7 \pm 25.4\end{array}$ & $\begin{array}{c}200-410 \\
316.7 \pm 61.9\end{array}$ & $<0.001^{*}$ \\
\hline $\operatorname{INR}(\%)$ & $\begin{array}{l}\text { Range } \\
\text { Mean } \pm S D\end{array}$ & $\begin{array}{l}1.0-1.2 \\
1.1 \pm 0.0\end{array}$ & $\begin{array}{l}1.0-1.2 \\
1.1 \pm 0.0\end{array}$ & 0.593 \\
\hline Serum Creatinine (mg/dl) & $\begin{array}{l}\text { Range } \\
\text { Mean } \pm S D\end{array}$ & $\begin{array}{l}5.7-8.2 \\
6.8 \pm 0.7\end{array}$ & $\begin{array}{l}0.7-1.3 \\
0.9 \pm 0.2\end{array}$ & $<0.001^{\star}$ \\
\hline Serum Urea $(\mathrm{mg} / \mathrm{dl})$ & $\begin{array}{l}\text { Range } \\
\text { Mean } \pm S D\end{array}$ & $\begin{array}{c}100-138 \\
119.9 \pm 9.7\end{array}$ & $\begin{array}{c}20-29 \\
23.7 \pm 2.6\end{array}$ & $<0.001^{\star}$ \\
\hline $\mathrm{K}^{+}(\mathrm{mmol} / \mathrm{L})$ & $\begin{array}{l}\text { Range } \\
\text { Mean } \pm S D\end{array}$ & $\begin{array}{l}3.3-5.1 \\
4.1 \pm 0.5\end{array}$ & $\begin{array}{l}3.3-4.9 \\
4.1 \pm 0.5\end{array}$ & 0.697 \\
\hline $\mathrm{Ca}^{+}(\mathrm{mmol} / \mathrm{L})$ & $\begin{array}{l}\text { Range } \\
\text { Mean } \pm S D\end{array}$ & $\begin{array}{l}1.0-1.2 \\
1.1 \pm 0.0\end{array}$ & $\begin{array}{l}1.0-1.2 \\
1.1 \pm 0.0\end{array}$ & 0.855 \\
\hline Albumin (gm/dl) & $\begin{array}{l}\text { Range } \\
\text { Mean } \pm S D\end{array}$ & $\begin{array}{l}3.0-3.8 \\
3.3 \pm 0.2\end{array}$ & $\begin{array}{l}4.0-4.8 \\
4.4 \pm 0.2\end{array}$ & $<0.001^{\star}$ \\
\hline HbAlc (\%) & $\begin{array}{l}\text { Range } \\
\text { Mean } \pm S D\end{array}$ & $\begin{array}{l}5.2-11.4 \\
6.9 \pm 1.5\end{array}$ & $\begin{array}{l}4.0-5.5 \\
4.7 \pm 0.5\end{array}$ & $<0.001^{\star}$ \\
\hline GA (\%) & $\begin{array}{l}\text { Range } \\
\text { Mean } \pm S D\end{array}$ & $\begin{array}{l}2.2-5.9 \\
3.6 \pm 0.9\end{array}$ & $\begin{array}{l}0.6-1.7 \\
1.2 \pm 0.3\end{array}$ & $<0.001^{\star}$ \\
\hline Fasting insulin $(\mu \mathrm{U} / \mathrm{ml})$ & $\begin{array}{l}\text { Range } \\
\text { Mean } \pm S D\end{array}$ & $\begin{array}{c}7.5-15 \\
11.1 \pm 1.8\end{array}$ & $\begin{array}{l}3.6-5.3 \\
4.5 \pm 0.4\end{array}$ & $<0.001^{\star}$ \\
\hline FBS (mg/dl) & Median (IQR) & $140(118.0-160.8)$ & $89.5(85-92.8)$ & $<0.001^{*}$ \\
\hline HOMA-IR & Median (IQR) & $4(3.0-4.9)$ & $1(1.0-1.0)$ & $<0.001^{*}$ \\
\hline
\end{tabular}

Abbreviations: BMI - body mass index; DM - diabetes mellitus; FBS - fasting blood sugar; GA - glycated albumin; Hb - hemoglobin; HbA1c - glycosylated hemoglobin; HOMA-IR - homeostatic model assessment of insulin resistance; INR - international normalized ratio; TLC - total leukocytic count. Statistical analysis: Independent samples t-test for parametric quantitative data between the two groups; Mann-Whitney test for non-parametric quantitative data (expressed by median and IQR) between the two groups; Chi square test (if expected values within cell $>5$ ) and Fisher's exact test (if expected values within cell $<5$ ) for qualitative data between the two groups. ${ }^{*} \mathrm{p}<0.05$. 
Table 2

Comparison of patients' group data in the start of the study and after 6 months follow up of regular hemodialysis.

\begin{tabular}{|c|c|c|c|c|}
\hline Variables & & $\begin{array}{l}\text { Baseline study } \\
\text { Patients }(n=40)\end{array}$ & $\begin{array}{l}\text { Follow up study } \\
\text { Patients }(n=40)\end{array}$ & p-value \\
\hline BMI $\left(\mathrm{kg} / \mathrm{m}^{2}\right)$ & $\begin{array}{l}\text { Range } \\
\text { Mean } \pm S D\end{array}$ & $\begin{array}{l}0.3-38.0 \\
26.6 \pm 5.4\end{array}$ & $\begin{array}{c}22.6-38.0 \\
27.4 \pm 3.3\end{array}$ & 0.310 \\
\hline $\mathrm{Hb}(\mathrm{gm} / \mathrm{dl})$ & $\begin{array}{l}\text { Range } \\
\text { Mean } \pm S D\end{array}$ & $\begin{array}{c}7-11 \\
9.3 \pm 1.0\end{array}$ & $\begin{array}{c}7.3-11.0 \\
9.1 \pm 0.7\end{array}$ & 0.406 \\
\hline $\mathrm{MCV}$ & $\begin{array}{l}\text { Range } \\
\text { Mean } \pm S D\end{array}$ & $\begin{array}{c}76-89 \\
83.0 \pm 3.4\end{array}$ & $\begin{array}{c}76-88 \\
81.0 \pm 2.6\end{array}$ & $0.008^{*}$ \\
\hline $\operatorname{TLC}\left(\times 10^{3}\right)$ & Median (IQR) & $\begin{array}{c}7\left(\mathrm{x} 10^{3}\right) \\
(5.9-9.0)\end{array}$ & $\begin{array}{l}6.9\left(\times 10^{3}\right) \\
(5.9-10.8)\end{array}$ & 0.939 \\
\hline Platelets $\left(\times 10^{3}\right)$ & $\begin{array}{l}\text { Range } \\
\text { Mean } \pm S D\end{array}$ & $\begin{array}{c}120-254 \\
166.7 \pm 25.4\end{array}$ & $\begin{array}{c}144-290 \\
196.2 \pm 40\end{array}$ & $<0.001^{\star}$ \\
\hline Serum Creatinine $(\mathrm{mg} / \mathrm{dl})$ & $\begin{array}{l}\text { Range } \\
\text { Mean } \pm S D\end{array}$ & $\begin{array}{l}5.7-8.2 \\
6.8 \pm 0.7\end{array}$ & $\begin{array}{l}5.3-8.4 \\
6.6 \pm 0.8\end{array}$ & 0.515 \\
\hline Serum Urea (mg/dl) & $\begin{array}{l}\text { Range } \\
\text { Mean } \pm S D\end{array}$ & $\begin{array}{c}100-138 \\
119.9 \pm 9.7\end{array}$ & $\begin{array}{c}67-134 \\
102 \pm 15.3\end{array}$ & $<0.001^{\star}$ \\
\hline URR (\%) & $\begin{array}{l}\text { Range } \\
\text { Mean } \pm S D\end{array}$ & $\begin{array}{c}60-68 \\
64.1 \pm 1.6\end{array}$ & $\begin{array}{c}63-72 \\
66.6 \pm 2.5\end{array}$ & $<0.001^{\star}$ \\
\hline $\mathrm{K}^{+}(\mathrm{mmol} / \mathrm{L})$ & $\begin{array}{l}\text { Range } \\
\text { Mean } \pm S D\end{array}$ & $\begin{array}{l}3.3-5.1 \\
4.1 \pm 0.5\end{array}$ & $\begin{array}{l}3.3-5.1 \\
4.2 \pm 0.5\end{array}$ & 0.571 \\
\hline $\mathrm{Ca}^{+}(\mathrm{mg} / \mathrm{dl})$ & $\begin{array}{l}\text { Range } \\
\text { Mean } \pm S D\end{array}$ & $\begin{array}{l}1.0-1.2) \\
1.1 \pm 0.0\end{array}$ & $\begin{array}{l}1.0-1.8 \\
1.1 \pm 0.1\end{array}$ & 0.440 \\
\hline Albumin (gm/dl) & $\begin{array}{l}\text { Range } \\
\text { Mean } \pm S D\end{array}$ & $\begin{array}{l}3.0-3.8 \\
3.3 \pm 0.2\end{array}$ & $\begin{array}{l}3.0-3.9 \\
3.5 \pm 0.2\end{array}$ & $0.040^{*}$ \\
\hline HbAlc $(\%)$ & $\begin{array}{l}\text { Range } \\
\text { Mean } \pm S D\end{array}$ & $\begin{array}{l}5.2-11.4 \\
6.9 \pm 1.5\end{array}$ & $\begin{array}{l}3.3-9.6 \\
5.6 \pm 1.5\end{array}$ & $<0.001^{*}$ \\
\hline GA (\%) & $\begin{array}{l}\text { Range } \\
\text { Mean } \pm S D\end{array}$ & $\begin{array}{l}2.2-5.9 \\
3.6 \pm 0.9\end{array}$ & $\begin{array}{l}1.1-4.2 \\
2.8 \pm 0.8\end{array}$ & $<0.001^{\star}$ \\
\hline Fasting insulin $(\mu \mathrm{U} / \mathrm{ml})$ & $\begin{array}{l}\text { Range } \\
\text { Mean } \pm S D\end{array}$ & $\begin{array}{l}7.5-15.0 \\
11.1 \pm 1.8\end{array}$ & $\begin{array}{c}10-15 \\
12.2 \pm 1.4\end{array}$ & $0.002^{*}$ \\
\hline FBS (mg/dl) & Median (IQR) & $140(118-160.8)$ & $130(110-156.5)$ & 0.237 \\
\hline HOMA-IR & Median (IQR) & $4(3.0-4.9)$ & $4(3.3-4.5)$ & 0.766 \\
\hline
\end{tabular}

Abbreviations: BMI - body mass index; FBS - fasting blood sugar; GA - glycated albumin; $\mathrm{Hb}$ hemoglobin; HbAlc - glycosylated hemoglobin; HOMA-IR - homeostatic model assessment of insulin resistance; MCV - mean cell volume; TLC - total leukocytic count; URR - urea reduction ratio. Statistical analysis: Paired samples t-test for parametric quantitative data between the two different times.

postprandial plasma glucose level, while $\mathrm{HbAlc} \%$ is closely associated with the fasting plasma glucose level (Dozio et al. 2018).

In the current study, according to baseline data and after 6-month follow-up, in the whole patients' group (40 patients) both HbAlc and GA were significantly lower than that at the start of study $(\mathrm{p}<0.001)$. URR $(\mathrm{p}<0.001)$ and serum albumin $(\mathrm{p}<0.04)$ were significantly lower at base time than after 6-month follow-up with $(\mathrm{p}<0.001)$. Sany et al. (2013) have demonstrated that $\mathrm{HbAlc}$ significantly underestimates glycemic control in diabetic patients on dialysis, relative to GA. In those with ESRD, lower HbA1c values were also associated with lower hemoglobin concentration and higher doses of erythropoietin (Sany et al. 2013). A limitation of the GA assay is found. In patients who are maintained on dialysis, albumin turnover should change and in patients who have chronic renal failure (CRF) with massive proteinuria, in whom GA values theoretically should be reduced as a result of shorter exposure to plasma albumin (Freedman 2012). After 6-month follow-up, HbAlc\%, GA\% and HOMA-IR were significantly lower in the controlled group than the uncontrolled group ( $<<0.023, p<0.015, p<0.001$, respectively). Urea level ( $\mathrm{p}=0.011), \mathrm{HbA1c} \%(\mathrm{p}=0.011), \mathrm{GA}(\mathrm{p}=0.001)$ and 
fasting insulin $(p=0.009)$ were significantly higher in the uncontrolled patients at start of study than after 6-month follow-up. URR at start of study was significantly lower than in the follow-up data with $(\mathrm{p}<0.002)$. Although HbAlc and GA reflect glycemic control during the preceding 4-6 and 1-2 weeks, respectively. The stable glycemic control during the preceding months and the correlation coefficient between plasma glucose (PG) and $\mathrm{HbA1c} \%$, which was similar with that between PG and GA, can negate the different impact of acute changes of glycemic control between $\mathrm{HbAlc} \%$ and GA. The measurement of $\mathrm{HbAlc} \%$ would result in an underestimation of glycemic control in HD patients with diabetes (Koga et al. 2017; Fukami et al. 2015).

In the present study, ROC curve analysis of HOMA-IR, HbAlc\% and GA\% for prediction of uncontrolled DM revealed that at optimal cut-off values $\geq 3.4, \geq 4.6$ and $\geq 2.0$, respectively, showed sensitivity $100 \%, 86.36 \%$ and $90.91 \%$; specificity $66.67 \%$, $61.11 \%$ and $50 \%$; accuracy $85 \%, 75 \%$ and $72.5 \%$ with AUC 0.831, 0.768 and 0.730. Buell et al. (2007) have found that $\mathrm{HbAlc} \%$ of $5.8 \%$ or greater is the point that yielded the highest specificity (92\%) and sensitivity (86\%). Moreover, they found that GA of $>231 \mu \mathrm{mol} / \mathrm{L}$ or greater provides specificity (70\%) and sensitivity

Table 3

Comparison of laboratory data (at start of study) between controlled and uncontrolled patient subgroups

\begin{tabular}{|c|c|c|c|c|}
\hline Baseline variables & & $\begin{array}{l}\text { Controlled DM } \\
\text { patients }(n=18)\end{array}$ & $\begin{array}{c}\text { Uncontrolled DM } \\
\text { patients }(n=22)\end{array}$ & p-value \\
\hline $\mathrm{Hb}(\mathrm{gm} / \mathrm{dl})$ & $\begin{array}{l}\text { Range } \\
\text { Mean } \pm \text { SD }\end{array}$ & $\begin{array}{c}(7.5-11.0) \\
9.3 \pm 1.0\end{array}$ & $\begin{array}{l}(7-11) \\
9.3 \pm 1.1\end{array}$ & 0.972 \\
\hline $\operatorname{TLC}\left(\times 10^{9} / \mathrm{L}\right)$ & Median (IQR) & $\begin{array}{c}6.7 \times 10^{9} / \mathrm{L} \\
(4.6-8.0)\end{array}$ & $\begin{array}{l}7.4 \times 10^{9} / \mathrm{L} \\
(6.4-13.1)\end{array}$ & $0.030^{*}$ \\
\hline Platelets $\left(\times 10^{9} / \mathrm{L}\right)$ & $\begin{array}{l}\text { Range } \\
\text { Mean } \pm S D\end{array}$ & $\begin{array}{c}(130-254) \\
168.2 \pm 28.8\end{array}$ & $\begin{array}{l}(120-208) \\
165.5 \pm 22.8\end{array}$ & 0.741 \\
\hline INR (\%) & $\begin{array}{l}\text { Range } \\
\text { Mean } \pm \text { SD }\end{array}$ & $\begin{array}{c}(1.0-1.2) \\
1.1 \pm 0.0\end{array}$ & $\begin{array}{c}(1.0-1.2) \\
1.1 \pm 0.0\end{array}$ & 0.234 \\
\hline Creatinine (mg/dl) & $\begin{array}{l}\text { Range } \\
\text { Mean } \pm S D\end{array}$ & $\begin{array}{c}(5.7-8.2) \\
6.6 \pm 0.7\end{array}$ & $\begin{array}{c}(5.7-8.2) \\
6.9 \pm 0.7\end{array}$ & 0.307 \\
\hline Urea $(\mathrm{mg} / \mathrm{dl})$ & $\begin{array}{l}\text { Range } \\
\text { Mean } \pm S D\end{array}$ & $\begin{array}{c}(102-138) \\
121.1 \pm 10.5\end{array}$ & $\begin{array}{l}(100-135) \\
119.0 \pm 9.1\end{array}$ & 0.521 \\
\hline URR (\%) & $\begin{array}{l}\text { Range } \\
\text { Mean } \pm S D\end{array}$ & $\begin{array}{c}(62-66) \\
64.1 \pm 1.4\end{array}$ & $\begin{array}{c}(60-68) \\
64 \pm 1.8\end{array}$ & 0.899 \\
\hline $\mathrm{K}^{+}(\mathrm{mmol} / \mathrm{L})$ & $\begin{array}{l}\text { Range } \\
\text { Mean } \pm S D\end{array}$ & $\begin{array}{c}(3.3-4.9) \\
4.1 \pm 0.5\end{array}$ & $\begin{array}{c}(3.4-5.1) \\
4.2 \pm 0.5\end{array}$ & 0.416 \\
\hline $\mathrm{Ca}^{+}(\mathrm{mmol} / \mathrm{L})$ & $\begin{array}{l}\text { Range } \\
\text { Mean } \pm S D\end{array}$ & $\begin{array}{c}(1.0-1.2) \\
1.1 \pm 0.1\end{array}$ & $\begin{array}{c}(1.0-1.2) \\
1.1 \pm 0.0\end{array}$ & 0.711 \\
\hline Albumin (gm/dl) & $\begin{array}{l}\text { Range } \\
\text { Mean } \pm S D\end{array}$ & $\begin{array}{c}(3.1-3.8) \\
3.3 \pm 0.2\end{array}$ & $\begin{array}{c}(3.0-3.7) \\
3.4 \pm 0.2\end{array}$ & 0.870 \\
\hline HbAlc (\%) & $\begin{array}{l}\text { Range } \\
\text { Mean } \pm S D\end{array}$ & $\begin{array}{c}(5.2-6.4) \\
6.0 \pm 0.5\end{array}$ & $\begin{array}{c}(6.2-11.0) \\
7.3 \pm 1.5\end{array}$ & 0.001 \\
\hline GA (\%) & $\begin{array}{l}\text { Range } \\
\text { Mean } \pm S D\end{array}$ & $\begin{array}{c}(2.2-5.1) \\
3.4 \pm 0.7\end{array}$ & $\begin{array}{c}(2.3-5.9) \\
3.8 \pm 0.9\end{array}$ & 0.171 \\
\hline Fasting insulin $(\mu \mathrm{U} / \mathrm{ml})$ & $\begin{array}{l}\text { Range } \\
\text { Mean } \pm S D\end{array}$ & $\begin{array}{c}(8-15) \\
11.2 \pm 1.8\end{array}$ & $\begin{array}{c}(7.5-14.0) \\
11 \pm 1.8\end{array}$ & 0.764 \\
\hline FBS (mg/dl) & Median (IQR) & $\begin{array}{c}128 \\
(117.0-148.5)\end{array}$ & $\begin{array}{c}150 \\
(119.5-188.3)\end{array}$ & 0.097 \\
\hline HOMA IR & Median (IQR) & $3.4(3.0-4.4)$ & $4.3(3.1-5.1)$ & 0.145 \\
\hline
\end{tabular}

Abbreviations: DM - diabetes mellitus; FBS - fasting blood sugar; GA - glycated albumin; $\mathrm{Hb}$ hemoglobin; HbA1c - glycosylated hemoglobin; HOMA-IR - homeostatic model assessment of insulin resistance; INR - international normalized ratio; TLC - total leukocytic count; URR - urea reduction ratio. Statistical analysis: Independent samples t-test for parametric quantitative data between the two groups; Mann Whitney test for non-parametric quantitative data (expressed by median and IQR) between the two groups. 
$(80 \%)$ as a screening test for diabetes and positive predictive value test of $75 \%$ and negative predictive value test of $85 \%$ (Furusyo et al. 2011). Freedman (2012) has concluded that HbAlc levels significantly underestimate glycemic control in patients on HD, while GA more accurately reflects recent control. The GA reference range (mean $\pm 2 \mathrm{SD}$ ) in Americans with normal glucose tolerance is $11.9-15.8 \%$. GA can be impacted by cirrhosis, albuminuria, smoking, and thyroid dysfunction (Hayashi et al. 2016). Meyer et al. (2013) have concluded that GA reflected glycemic control more accurately and this supports its potential as a marker of choice. Cefalu et al. (2017) have found that GA with a cut-off value of $250 \mu \mathrm{mol} / \mathrm{L}$ has the sensitivity to detect diabetes ( $81 \%)$, specificity $(87 \%)$ and positive predictive value (43\%). Kobayashi et al. (2016) have identified GA as the only independent factor associated with post-prandial plasma glucose. GA was not significantly associated with protein loss because albumin synthesis in the liver made up for the loss in the urine and PD fluid. Therefore, GA might be useful for checking glycemic control in PD patients with no albumin loss. Divani et al. (2018) have concluded that GA is a stronger indicator of

Table 4

Comparison of laboratory data after 6 months follow up between diabetic patients' subgroups

\begin{tabular}{|c|c|c|c|c|}
\hline $\begin{array}{l}\text { After } 6 \text { months follow up } \\
\text { variables }\end{array}$ & & $\begin{array}{c}\text { Controlled DM } \\
\text { subgroups }(n=18)\end{array}$ & $\begin{array}{l}\text { Uncontrolled DM } \\
\text { subgroups }(n=22)\end{array}$ & p-value \\
\hline $\mathrm{Hb}(\mathrm{gm} / \mathrm{dl})$ & $\begin{array}{l}\text { Range } \\
\text { Mean } \pm S D\end{array}$ & $\begin{array}{c}(7.3-11.0) \\
9 \pm 0.9\end{array}$ & $\begin{array}{c}(8.4-10.2) \\
9.2 \pm 0.6\end{array}$ & 0.476 \\
\hline $\operatorname{TLC}\left(\times 10^{9} / \mathrm{L}\right)$ & Median (IQR) & $6.3(4.9-7.3)$ & $9.5(6.2-13.2)$ & $0.007^{\star}$ \\
\hline Platelets $\left(\times 10^{9} / \mathrm{L}\right)$ & $\begin{array}{l}\text { Range } \\
\text { Mean } \pm S D\end{array}$ & $\begin{array}{l}(156-266) \\
194.4 \pm 32.0\end{array}$ & $\begin{array}{c}(144-290) \\
197.7 \pm 46.2\end{array}$ & 0.797 \\
\hline INR (\%) & $\begin{array}{l}\text { Range } \\
\text { Mean } \pm S D\end{array}$ & $\begin{array}{c}(1.0-1.2) \\
1.1 \pm 0.0\end{array}$ & $\begin{array}{c}(1.0-1.2) \\
1.1 \pm 0.1\end{array}$ & 0.485 \\
\hline Creatinine (mg/dl) & $\begin{array}{l}\text { Range } \\
\text { Mean } \pm S D\end{array}$ & $\begin{array}{c}(5.7-8.2) \\
6.8 \pm 0.9\end{array}$ & $\begin{array}{c}(5.3-8.4) \\
6.5 \pm 0.8\end{array}$ & 0.178 \\
\hline Urea (mg/dl) & $\begin{array}{l}\text { Range } \\
\text { Mean } \pm S D\end{array}$ & $\begin{array}{c}(85-134) \\
108.4 \pm 15.1\end{array}$ & $\begin{array}{c}(67-120) \\
96.9 \pm 13.8\end{array}$ & $0.016^{*}$ \\
\hline URR (\%) & $\begin{array}{l}\text { Range } \\
\text { Mean } \pm S D\end{array}$ & $\begin{array}{l}(63-72) \\
67.2 \pm 2.4\end{array}$ & $\begin{array}{l}(63-72) \\
66.1 \pm 2.5\end{array}$ & 0.169 \\
\hline $\mathrm{K}^{+}(\mathrm{mmol} / \mathrm{L})$ & $\begin{array}{l}\text { Range } \\
\text { Mean } \pm S D\end{array}$ & $\begin{array}{c}(3.4-4.7) \\
4.1 \pm 0.4\end{array}$ & $\begin{array}{l}(3.3-5.1) \\
4.3 \pm 0.6\end{array}$ & 0.356 \\
\hline $\mathrm{Ca}^{+}(\mathrm{mmol} / \mathrm{L})$ & $\begin{array}{l}\text { Range } \\
\text { Mean } \pm S D\end{array}$ & $\begin{array}{c}(1.0-1.2) \\
1.1 \pm 0.1\end{array}$ & $\begin{array}{c}(1.0-1.8) \\
1.1 \pm 0.2\end{array}$ & 0.391 \\
\hline Albumin (gm/dl) & $\begin{array}{l}\text { Range } \\
\text { Mean } \pm S D\end{array}$ & $\begin{array}{c}(3.1-3.9) \\
3.5 \pm 0.2\end{array}$ & $\begin{array}{c}(3.0-3.8) \\
3.4 \pm 0.2\end{array}$ & 0.533 \\
\hline $\operatorname{HbAlc}(\%)$ & $\begin{array}{l}\text { Range } \\
\text { Mean } \pm S D\end{array}$ & $\begin{array}{c}(3.3-8.1) \\
5.0 \pm 1.5\end{array}$ & $\begin{array}{c}(4.3-9.6) \\
6.1 \pm 1.5\end{array}$ & $0.023^{\star}$ \\
\hline GA (\%) & $\begin{array}{l}\text { Range } \\
\text { Mean } \pm S D\end{array}$ & $\begin{array}{c}(1.1-4.1) \\
2.3 \pm 0.8\end{array}$ & $\begin{array}{c}(1.6-4.2) \\
2.9 \pm 0.7\end{array}$ & $0.015^{\star}$ \\
\hline Fasting insulin $(\mu \mathrm{U} / \mathrm{ml})$ & $\begin{array}{l}\text { Range } \\
\text { Mean } \pm S D\end{array}$ & $\begin{array}{c}(10-14) \\
11.8 \pm 1.4\end{array}$ & $\begin{array}{c}(11-15) \\
12.5 \pm 1.3\end{array}$ & 0.094 \\
\hline FBS (mg/dl) & Median (IQR) & $\begin{array}{c}110 \\
(100.0-140.5)\end{array}$ & $\begin{array}{c}150 \\
(126.8-160.8)\end{array}$ & $0.007^{\star}$ \\
\hline HOMA-IR & Median (IQR) & $3.2(2.9-3.8)$ & $4.4(3.9-4.9)$ & $<0.001^{\star}$ \\
\hline $\mathrm{T}-\mathrm{Ch}(\mathrm{mg} / \mathrm{dl})$ & Median (IQR) & $\begin{array}{c}195 \\
(132.5-240.0)\end{array}$ & $\begin{array}{c}227.5 \\
(209.8-273.3)\end{array}$ & $0.027^{\star}$ \\
\hline $\mathrm{TG}(\mathrm{mg} / \mathrm{dl})$ & Median (IQR) & $\begin{array}{c}144.5 \\
(114.8-171.5) \\
\end{array}$ & $\begin{array}{c}145 \\
(120-245)\end{array}$ & 0.488 \\
\hline
\end{tabular}

Abbreviations: DM - diabetes mellitus; FBS - fasting blood sugar; GA - glycated albumin; Hb hemoglobin; HbA1c - glycosylated hemoglobin; HOMA-IR - homeostatic model assessment of insulin resistance; INR - international normalized ratio; TG - triglycerides; TLC - total leukocytic count; URR - urea reduction ratio. Statistical analysis: Independent samples t-test for parametric quantitative data between the two groups; Mann Whitney test for non-parametric quantitative data (expressed by median and IQR) between the two groups. 
Table 5

Correlation between clinical, laboratory data, and glycemic markers in the study group in start of the study

\begin{tabular}{|c|c|c|c|c|}
\hline \multirow{2}{*}{$\begin{array}{l}\text { Baseline variables } \\
\text { of the studied patients }(n=40)\end{array}$} & \multicolumn{2}{|c|}{ HbAlc\% } & \multicolumn{2}{|c|}{ GA\% } \\
\hline & $\mathbf{r}$ & p-value & $\mathbf{r}$ & p-value \\
\hline HBAlc $(\%)$ & & & 0.656 & $<0.001^{\star}$ \\
\hline GA (\%) & 0.656 & $<0.001^{*}$ & & \\
\hline DM duration (Years) & -0.017 & 0.919 & 0.067 & 0.680 \\
\hline HTN duration (Years) & -0.240 & 0.248 & 0.106 & 0.613 \\
\hline Erythropoietin dosage & -0.274 & 0.087 & -0.119 & 0.466 \\
\hline HD duration (months) & -0.052 & 0.749 & -0.013 & 0.938 \\
\hline URR (\%) & 0.090 & 0.582 & -0.143 & 0.377 \\
\hline BMI $\left(\mathrm{kg} / \mathrm{m}^{2}\right)$ & -0.187 & 0.248 & -0.055 & 0.734 \\
\hline $\mathrm{Hb}(\mathrm{gm} / \mathrm{dl})$ & 0.357 & $0.024^{\star}$ & 0.062 & 0.706 \\
\hline TLC $\left(\times 10^{9} / \mathrm{L}\right)$ & 0.017 & 0.917 & -0.239 & 0.137 \\
\hline Platelets counts $\left(\times 10^{9} / \mathrm{L}\right)$ & 0.092 & 0.571 & 0.042 & 0.795 \\
\hline INR (\%) & -0.017 & 0.917 & -0.074 & 0.648 \\
\hline Creatinine $(\mathrm{mg} / \mathrm{dl})$ & 0.195 & 0.227 & 0.081 & 0.618 \\
\hline Urea (mg/dl) & -0.092 & 0.574 & -0.135 & 0.405 \\
\hline $\mathrm{K}^{+}(\mathrm{mmol} / \mathrm{L})$ & 0.167 & 0.304 & 0.018 & 0.914 \\
\hline $\mathrm{Ca}^{+}(\mathrm{mg} / \mathrm{dl})$ & -0.069 & 0.674 & -0.160 & 0.325 \\
\hline Serum albumin(gm/dl) & 0.183 & 0.258 & 0.149 & 0.360 \\
\hline Fasting insulin $(\mu \mathrm{U} / \mathrm{ml})$ & 0.116 & 0.476 & -0.117 & 0.474 \\
\hline FBS (mg/dl) & 0.943 & $<0.001^{\star}$ & 0.664 & $<0.001^{\star}$ \\
\hline HOMA-IR & 0.875 & $<0.001^{\star}$ & 0.535 & $<0.001^{\star}$ \\
\hline $\mathrm{TC}(\mathrm{mg} / \mathrm{dl})$ & 0.705 & $<0.001^{*}$ & 0.563 & $<0.001^{\star}$ \\
\hline $\mathrm{TG}(\mathrm{mg} / \mathrm{dl})$ & 0.416 & $0.008^{*}$ & 0.294 & 0.065 \\
\hline
\end{tabular}

Abbreviations: BMI - body mass index; DM - diabetes mellitus; FBS - fasting blood sugar; GA glycated albumin; Hb - hemoglobin; HbAlc - glycosylated hemoglobin; HD - hemodialysis; HOMAIR - homeostatic model assessment of insulin resistance; HTN - hypertension; INR - international normalized ratio; TC - total cholesterol; TG - triglycerides; TLC - total leukocytic count; URR - urea reduction ratio. Statistical analysis: Pearson's correlation; ${ }^{*} \mathrm{p}<0.05$.

poor glycemic control when compared to HbAlc\% among diabetic HD patients.

In the current study, levels of both HbAlc and GA showed highly significant correlation between each of them and fasting blood sugar (FBS), hemoglobin $(\mathrm{p}<0.024)$, total cholesterol, HOMA-IR $(\mathrm{p}<0.001)$ and triglycerides $(\mathrm{p}<0.008)$. In the study of Hayashi et al. (2016), HbAlc\% correlated with the average glucose more than GA\%, but underestimates it. It needs a development of a correction formula of $\mathrm{HbAlc} \%$ as an acceptable marker. GA\% value reflects the average glucose and although it is less accurate than $\mathrm{HbAlc} \%$ it could serve as an indicator for hyperglycemia/hypoglycemia excursion (Hayashi et al. 2016). Fukami et al. (2015) have suggested that adjusted GA might be a better indicator of glycemic control than measured $\mathrm{HbAlc} \%$. Adjusted GA may be a better indicator than GA for evaluating the glycemic control in type-2 DM patients with short duration of HD (ADA 2018).

In the current study, GA\% and $\mathrm{HbAlc} \%$ showed significant correlation with total leukocytic count $(\mathrm{p}<0.034$ and $\mathrm{p}<0.003)$, respectively. The total leukocytic count showed highly significant association for prediction of the diabetic complications $(\mathrm{p}=0.007)$.

The increase in GA levels is associated with increased oxidative stress, endothelial dysfunction, and pro-inflammatory responses. This might 
Table 6

Correlation between laboratory data and glycemic markers in the patients' group after 6 months follow up

\begin{tabular}{|c|c|c|c|c|}
\hline \multirow{2}{*}{$\begin{array}{l}6 \text { months follow up of whole } \\
\text { patients' group }(n=40)\end{array}$} & \multicolumn{2}{|c|}{ HbAlc\% } & \multicolumn{2}{|c|}{ GA\% } \\
\hline & $\mathbf{r}$ & p-value & $\mathbf{r}$ & p-value \\
\hline HbA1c (\%) & & & 0.693 & $<0.001^{\star}$ \\
\hline GA $(\%)$ & 0.693 & $<0.001^{\star}$ & & \\
\hline DM duration (Years) & -0.177 & 0.274 & -0.267 & 0.096 \\
\hline HTN duration (Years) & -0.187 & 0.370 & -0.006 & 0.979 \\
\hline Erythropoietin dosage & -0.155 & 0.338 & 0.010 & 0.953 \\
\hline HD duration & -0.214 & 0.186 & -0.159 & 0.327 \\
\hline URR (\%) & -0.264 & 0.100 & 0.084 & 0.607 \\
\hline BMI $\left(\mathrm{kg} / \mathrm{m}^{2}\right)$ & -0.032 & 0.842 & 0.183 & 0.258 \\
\hline $\mathrm{Hb}(\mathrm{gm} / \mathrm{dl})$ & 0.556 & $<0.001^{\star}$ & 0.413 & $0.008^{\star}$ \\
\hline TLC $\left(\times 10^{9} / \mathrm{L}\right)$ & 0.462 & $0.003^{*}$ & 0.336 & $0.034^{*}$ \\
\hline Platelets $\left(\times 10^{9} / \mathrm{L}\right)$ & -0.128 & 0.430 & -0.233 & 0.147 \\
\hline INR (\%) & -0.166 & 0.306 & -0.140 & 0.390 \\
\hline Creatinine (mg/dl) & 0.009 & 0.957 & 0.185 & 0.254 \\
\hline Urea (mg/dl) & 0 & 0.999 & -0.016 & 0.923 \\
\hline $\mathrm{K}^{+}(\mathrm{mmol} / \mathrm{L})$ & 0.175 & 0.281 & 0.134 & 0.410 \\
\hline $\mathrm{Ca}^{+}(\mathrm{mmol} / \mathrm{L})$ & -0.136 & 0.403 & 0.023 & 0.886 \\
\hline Serum albumin $(\mathrm{gm} / \mathrm{dl})$ & 0.065 & 0.692 & 0.151 & 0.352 \\
\hline Fasting insulin level $(\mu \mathrm{U} / \mathrm{ml})$ & 0.918 & $<0.001^{\star}$ & 0.658 & $<0.001^{\star}$ \\
\hline FBS (mg/dl) & -0.138 & 0.396 & -0.152 & 0.349 \\
\hline HOMA-IR & 0.895 & $<0.001^{\star}$ & 0.622 & $<0.001^{\star}$ \\
\hline TC (mg/dl) & 0.227 & 0.159 & 0.358 & $0.023^{*}$ \\
\hline $\mathrm{TG}(\mathrm{mg} / \mathrm{dl})$ & 0.269 & 0.093 & 0.166 & 0.305 \\
\hline
\end{tabular}

Abbreviations: BMI - body mass index; DM - diabetes mellitus; FBS - fasting blood sugar; GA glycated albumin; $\mathrm{Hb}$ - hemoglobin; HbA1c - glycosylated hemoglobin; HD - hemodialysis; HOMAIR - homeostatic model assessment of insulin resistance; HTN - hypertension; INR - international normalized ratio; TC - total cholesterol; TG - triglycerides; TLC - total leukocytic count; URR - urea reduction ratio. Statistical analysis: Pearson's correlation; ${ }^{*} \mathrm{p}<0.05$.

indicate that GA may play a role in the pathogenesis of vascular complications in diabetic patients with ESRD. Hence, GA is a good predictor of long-term survival in patients undergoing $\mathrm{HD}$, in addition is being a glucose control index (National Kidney Foundation 2012). Chen et al. (2017) have found that HD patients had lower HbAlc\% levels despite of the higher random glucose and GA levels. The ratio of $\mathrm{GA} / \mathrm{HbAlc}$ was increased in HD patients relative to patients without renal insufficiency. They have also observed that $\mathrm{HbAlc} \%$ correlated positively with hemoglobin and negatively with erythropoietin stimulating agents (Chen et al. 2017). Glycemic control improves spontaneously in some diabetic patients on dialysis, leading to normal HbA1c\% levels, known as "burnt-out diabetes." It has been found in $17.7 \%$ of patients with diabetes on dialysis based on $\mathrm{HbAlc} \%$, but when taking GA\% into account, the rate was significantly decreased to $10.9 \%$ (Peng et al. 2015).

The whole patients' group compared to the healthy control group had significantly higher BMI as the whole control group was selected to have normal BMI. Significant correlation was found between serum total cholesterol and GA\%. No significant correlation was found between serum albumin and HbAlc, GA and BMI, and HOMA. The current results agree with the results of earlier studies of Rhee et al. (2015) done on type-2 DM on hemodialysis. They found that 
poor glycemic control was further associated with components of metabolic syndrome including high lipid profile, higher BMI, uncontrolled $\mathrm{Bp}$, and with markers of malnutrition and inflammation. Inoue et al. (2015) have found that the BMI is known not to affect the HbA1c values, while a negative correlation exists between the BMI and GA. The reason for these relations is that obesity and chronic inflammatory reactions might increases albumin turnover (Inoue et al. 2015). Rhee et al. (2015) have concluded that higher albumin levels are associated with the improved glycemic control. They have suggested that poor glycemic control is associated with markers of an increased inflammation and infection, independent of BMI. They also have found that higher eGFR at initiation of dialysis was associated with poor glycemic control, suggesting that sicker patients in uremic state who initiate dialysis earlier may have higher $\mathrm{HbAlc} \%$ levels. Uremic toxins influence glucose homeostasis through increasing insulin resistance, promoting hepatic gluconeogenesis, and suppressing the utilization of peripheral glucose in ESRD patients (Rhee et al. 2015). Another explanation could be that patients with a higher eGFR have lower creatinine production considered as a marker for lower muscle mass and malnutrition which are known to be involved in the pathogenesis of diabetes.

Albuminuria falls with lower GFR in patients on dialysis, potentially minimizing the effect of albuminuria in ESRD. GA reflects the percentage of albumin that is glycated regardless of total concentration (Sany et al. 2013). On the other hand, Freedman (2012) has concluded that HbAlc \% is limited not only by advanced nephropathy, but it is also subjected to error from rapidly changing diabetes control, hemolytic anemia, severe anemia, recent blood transfusion, iron deficiency, erythropoietin or other drugs interacting with erythropoiesis. Despite the shorter half-life of glycemic control, GA measurements provide useful information to guide physicians in the care of patients with HD (Freedman 2012).

In the current study serum albumin, URR\%, erythropoietin doses did not show any significant correlation with both $\mathrm{HbA} 1 \mathrm{c} \%$ and GA\%. HbA1c\% correlated significantly with GA, HOMA-IR, fasting insulin level and hemoglobin concentration $(\mathrm{p}<0.001)$ after 6-month follow-up. GA had also the same significant correlations with serum total cholesterol $(\mathrm{p}<0.023)$. Inoue et al. (2015) have examined the correlation between $\mathrm{HbA1c} \%$ and GA\%, and established an equation $\left[\mathrm{HbA} 1 \mathrm{c} \%=0.216 \times \mathrm{GA}+2.978\left(\mathrm{R}^{2}=0.5882\right.\right.$, $\mathrm{p}<0.001)$ ], which made diabetes treatment based on a given GA value, instead of measuring $\mathrm{HbAlc} \%$, in
Table 7

Simple logistic regression analysis for prediction of uncontrolled DM $(n=40)$

\begin{tabular}{lccc}
\hline Variables & OR & $\mathbf{9 5 \%}$ CI & p-value \\
\hline TLC $\left(\mathrm{x} 10^{3}\right)$ & 1.56 & $1.13-2.16$ & $0.007^{*}$ \\
Urea $(\mathrm{mg} / \mathrm{dl})$ & 0.94 & $0.90-0.99$ & $0.025^{*}$ \\
\hline HbA1c $(\%)$ & 1.77 & $1.04-3.02$ & $0.035^{*}$ \\
GA $(\%)$ & 2.86 & $1.17-7.04$ & $0.022^{*}$ \\
FBS $(\mathrm{mg} / \mathrm{dl})$ & 1.02 & $0.99-1.04$ & 0.062 \\
HOMA-IR & 2.9 & $1.21-6.95$ & $0.017^{*}$ \\
T-Cholesterol $(\mathrm{mg} / \mathrm{dl})$ & 1.01 & $1.001-1.030$ & $0.028^{*}$ \\
\hline
\end{tabular}

Abbreviations: CI - Confidence Interval; FBS - fasting

blood sugar; GA - glycated albumin; HbAlc - glycosylated hemoglobin; HOMA-IR - homeostatic model assessment of insulin resistance; OR - Odds Ratio; TLC - total leukocytic count.

Table 8

ROC curve analysis for prediction of glucose homeostasis in diabetic patients $(\mathrm{n}=40)$

\begin{tabular}{lccc}
\hline Patients (n=40) & HbA1c (\%) & GA (\%) & HOMA-IR \\
\hline Optimal cut-off point & $\geq 4.6$ & $\geq 2.0$ & $\geq 3.4$ \\
AUC & 0.768 & 0.730 & 0.831 \\
95\% CI & $0.607-0.886$ & $0.566-0.858$ & $0.679-0.930$ \\
p-value & $<0.001^{*}$ & $0.006^{*}$ & $<0.001^{*}$ \\
Sensitivity & $86.36 \%$ & $90.91 \%$ & $100 \%$ \\
Specificity & $61.11 \%$ & $50.0 \%$ & $66.67 \%$ \\
Positive Predictive Value & $73.1 \%$ & $69 \%$ & $78.6 \%$ \\
Negative Predictive Value & $78.6 \%$ & $81.8 \%$ & $100 \%$ \\
Diagnostic Accuracy & $75 \%$ & $72.5 \%$ & $85 \%$ \\
\hline
\end{tabular}

Abbreviations: AUC - Area Under Curve; CI - Confidence Interval; GA - glycated albumin; HbA1c - glycosylated hemoglobin; HOMA-IR - homeostatic model assessment of insulin resistance; ROC - receiver operating characteristic. Statistical analysis: ${ }^{*} \mathrm{p}<0.05$.

patients whose blood control was not stable or with altered hemoglobin metabolism. Serum GA specifically measures albumin that has undergone glycation. It represents short-term (1-3 weeks) glycemia. It is not affected by anemia, and usage of erythropoietin (Inoue et al. 2015). The concentrations were closely correlated with the level of HbAlc (Inoue et al. 2015). Kobayashi et al. (2016), Dozio et al. (2018), and Abe et al. $(2018,2019)$ have concluded that GA is useful in monitoring the glucose homeostasis in type-2 DM patients maintained on HD. However, they reported that GA can be affected by protein loss. GA in 
patients undergoing online HDF or HD was practically reliable even if dialysis modality was changed. Both GA and HbAlc will be correlated with glycemic status, if albumin leakage is clinically acceptable and red blood cell lifespan is stable (Wallace et al. 2004).

As $\mathrm{HbAlc} \%$ is a long-term glycemic indicator, GA is a medium-term glycemic marker because it reflects the average life of albumin (about 20 days). This means GA can promptly indicate either an improvement or a worsening of the patient's glycemic status. Therefore, it is useful in conditions requiring short-term glycemic control, as after starting or modifying a drug therapy (National Kidney Foundation 2012). The cut-offs proposed for DM screening are GA $>15.2 \%$ or $>15.5 \%$ (Weykamp et al. 2004; Yajima et al. 2017).

\section{Conclusion}

The current study showed that GA\% is more sensitive than $\mathrm{HbAlc} \%$. (90.91\% vs $86.36 \%$ ) but less specific (50\% vs $61.11 \%$ ), respectively in follow-up of diabetes homeostasis in diabetic patients maintained on hemodialysis. Diagnostic accuracy is higher in HbAlc\% than in GA\% (75\% vs $72.5 \%$ ), respectively. HOMA-IR is superior for both as regards sensitivity $(100 \%)$ and diagnostic accuracy (85\%).

Our study design was limited by the small sample used. Additional long-term studies are needed to accurately assess the effectiveness of GA in dialysis patients. Follow-up also is needed in all types of renal replacement therapy to document the parameter of choice that is needed to monitor blood sugar in this group of patients.

\section{Acknowledgements}

We thank to the members of the Haemodialysis Unit and Clinical Pathology Department, Minia University Hospital, Egypt and Dr. Mostafa Assem who did the statistical analysis of the study.

\section{References}

Abe M, Hamano T, Hoshino J, Wada A, Nakai S, Masakane I. Rate of the "burnt-out diabetes" phenomenon in patients on peritoneal dialysis. Diabetes Res Clin Pract 143, 254-262, 2018.

Abe M, Hamano T, Hoshino J, Wada A, Nakai S, Masakane I. Glycemic control and survival in peritoneal dialysis patients with diabetes: A 2-year nationwide cohort study. Sci Rep 9, 3320, 2019.

American Diabetes Association. 1. Improving Care and Promoting Health in Populations: Standards of Medical Care in Diabetes-2018. Diabetes Care 41, S7-S12, 2018.

Aramwit P, Satirapoj B. Chapter 8. Glycemic control in diabetic patients on long-term maintenance dialysis. In Hemodialysis (Ed. Hiromichi Suzuki), InTechOpen Limited, London, United Kingdom, pp.153-191, 2013.

Buell C, Kermah D, Davidson MB. Utility of A1C for diabetes screening in the 1999-2004 NHANES population. Diabetes Care 30, 2233-2235, 2007.

Campbell AK. Detection and quantification of chemiluminescence. In Chemiluminescence principles and applications in biology and medicine (Ed. Ellis Horwood), Chichester, pp. 68-126, 1988.

Cefalu WT, Buse JB, Tuomilehto J, Fleming GA, Ferrannini E, Gerstein HC, Bennett PH, Ramachandran A, Raz I, Rosenstock J, Kahn SE. Response to Comment on Cefalu et al. Update and next steps for real-world translation of interventions for type 2 diabetes prevention: Reflections from a diabetes care Editors' Expert Forum. Diabetes Care 39, 1186-1201, 2016. Diabetes Care 40, e23-e24, 2017.

Chen CW, Drechsler C, Suntharalingam P, Karumanchi A Christoph Wanner C, Berg AH. High glycated albumin and mortality in persons with diabetes mellitus on hemodialysis. Clin Chem 63, 477-485, 2017.

Cohen MP, Hud E. Measurement of plasma glycoalbumin levels with a monoclonal antibody based ELISA. J Immunol Methods 122, 279-283, 1989.

Divani M, Georgianos PI, Didangelos T, Iliadis F, Makedou A, Hatzitolios A, Liakopoulos V, Grekas DM. Comparison of glycemic markers in chronic hemodialysis using continuous glucose monitoring. Am J Nephrol 47, $21-29,2018$

Dozio E, Corradi V, Proglio M, Vianello E, Menicanti L, Rigolini R, Caprara C, de Cal M, Corsi Romanelli MM, Ronco C. Usefulness of glycated albumin as a biomarker for glucose control and prognostic factor in chronic kidney disease patients on dialysis (CKD-G5D). Diabetes Res Clin Pract 140, 9-17, 2018.

Freedman BI. A critical evaluation of glycated protein parameters in advanced nephropathy: a matter of life or death: time to dispense with the hemoglobin A1C in end-stage kidney disease. Diabetes Care 35, 1621-1624, 2012.

Fukami K, Shibata R, Nakayama H, Yamada K, Okuda S, Koga M. Serum albumin-adjusted glycated albumin reflects glycaemic excursion in diabetic patients with severe chronic kidney disease not treated with dialysis. J Diabetes Complications 29, 913-917, 2015. 
Furusyo N, Koga T, Ai M, Otokozawa S, Kohzuma T, Ikezaki H, Schaefer EJ, Hayashi J. Utility of glycated albumin for the diagnosis of diabetes mellitus in a Japanese population study: results from the Kyushu and Okinawa Population Study (KOPS). Diabetologia 54, 3028-3036, 2011.

Hayashi A, Takano K, Masaki T, Yoshino S, Ogawa A, Shichiri M. Distinct biomarker roles for HbA1c and glycated albumin in patients with type 2 diabetes on hemodialysis. J Diabetes Complications 30, 1494-1499, 2016.

Inoue K, Goto A, Kishimoto M, Tsujimoto T, Yamamoto-Honda R, Noto H, Kajio H, Terauchi Y, Noda M. Possible discrepancy of HbAlc values and its assessment among patients with chronic renal failure, hemodialysis and other diseases. Clin Exp Nephrol 19, 1179-1183, 2015.

Kobayashi H, Abe M, Yoshida Y, Suzuki H, Maruyama N, Okada K. Glycated albumin versus glycated hemoglobin as a glycemic indicator in diabetic patients on peritoneal dialysis. Int J Mol Sci 17, 619, 2016.

Koga M, Inada S, Nakao T, Kawamori R, Kasayama S. The glycated albumin (GA) to HbAlc ratio reflects shorterterm glycemic control than GA: Analysis of patients with fulminant type 1 diabetes. J Clin Lab Anal 31, e22023, 2017.

Meyer L, Chantrel F, Imhoff O, Sissoko A, Serb L, Dorey F, Fleury D, Smagala A, Kepenekian L, Krummel T, Le Floch JP, Kessler L. Glycated albumin and continuous glucose monitoring to replace glycated haemoglobin in patients with diabetes treated with haemodialysis. Diabet Med 30, 1388-1389, 2013.

National Kidney Foundation. KDOQI Clinical Practice Guideline for Diabetes and CKD: 2012 Update. Am J Kidney Dis 60, 850-886, 2012.

Peng F, Xia X, He F, Li Z, Huang F, Yu X. The effect of glycated hemoglobin and albumin-corrected glycated serum protein on mortality in diabetic patients receiving continuous peritoneal dialysis. Perit Dial Int 35, 566-575, 2015.

Rhee JJ, Ding VY, Rehkopf DH, Arce CM, Winkelmayer WC. Correlates of poor glycemic control among patients with diabetes initiating hemodialysis for end-stage renal disease. BMC Nephrol 16, 204, 2015.

Sany D, Elshahawy Y, Anwar W. Glycated albumin versus glycated hemoglobin as glycemic indicator in hemodialysis patients with diabetes mellitus: Variables that influence. Saudi J Kidney Dis Transpl 24, 260-273, 2013.

Wallace TM, Levy JC, Matthews DR. Use and abuse of HOMA modeling. Diabetes Care 27, 1487-1495, 2004.

Weykamp C, John WG, Mosca A, Hoshino T, Little R, Jeppsson JO, Goodall I, Miedema K, Myers G, Reinauer H, Sacks DB, Slingerland R, Siebelder C. The IFCC reference measurement system for HbA1c: a 6-year progress report. Clin Chem 54, 240-248, 2008.

Yajima T, Yajima K, Hayashi M, Takahashi H, Yasuda K. Serum albumin-adjusted glycated albumin as a better indicator of glycemic control in Type 2 diabetes mellitus patients with short duration of hemodialysis. Diabetes Res Clin Pract 130, 148-153, 2017. 